\title{
DESEMPENHO E MORFOMETRIA DUODENAL DE FRANGOS DE CORTE SUBMETIDOS A DIFERENTES NÍVEIS DE ENERGIA E PROGRAMAS DE ALIMENTAÇÃO DE 42 A 57 DIAS DE IDADE
}

\author{
KARINA FERREIRA DUARTE ${ }^{1}$, OtTO MACK JUNQUeIRA ${ }^{2}$, LILIANA LONGO BORGES ${ }^{3}$, Elaine Talita

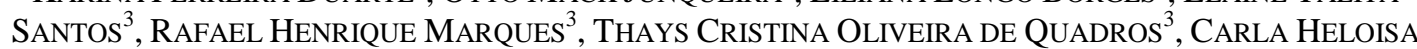 \\ DE FARIA DOMINGUES ${ }^{3}$
}

\begin{abstract}
${ }^{1}$ Pós-doutoranda da Faculdade de Ciências Agrárias e Veterinárias da Unesp, Jaboticabal, SP, Brasil. karinafduarte@ yahoo.com.br ${ }^{2}$ Professor Doutor da Faculdade de Ciências Agrárias e Veterinárias da Unesp, Jaboticabal, SP, Brasil.

${ }^{3}$ Pós-Graduandos da Faculdade de Ciências Agrárias e Veterinárias da Unesp, Jaboticabal, SP, Brasil.
\end{abstract}

\begin{abstract}
O experimento foi realizado com o objetivo de avaliar diferentes níveis de energia e programas de alimentação sobre o desempenho, as características de carcaça e a morfometria da mucosa do duodeno de frangos de corte de 42 a 57 dias de idade. Foram utilizados 1.600 pintos machos "Cobb 500", em um delineamento inteiramente ao acaso, em esquema fatorial $2 \mathrm{X} 4$, sendo dois níveis de energia (3.200 e $3.600 \mathrm{kcal} \mathrm{EM} / \mathrm{kg}$ ) e quatro programas de alimentação. Os níveis energéticos ou os diferentes programas de alimentação não determinaram diferenças

expressivas nas características de carcaça que justifiquem a escolha de um nível de energia ou de um programa alimentar, devendo prevalecer o nível energético e as recomendações de aminoácidos que determinem o maior custo-benefício. Observou-se que o nível $3.600 \mathrm{kcal}$ $\mathrm{EM} / \mathrm{kg}$ proporcionou melhoria no desempenho das aves e na morfometria da mucosa intestinal e o fracionamento das exigências de aminoácidos digestíveis em dois períodos piorou os resultados de desempenho e de altura de vilosidade.
\end{abstract}

PALAVRAS-CHAVE: Aminoácidos digestíveis; energia metabolizável; exigências nutricionais; intestino delgado.

\section{PERFORMANCE AND DUODENUM MORPHOMETRY OF BROILER CHICKENS SUBMITTED TO DIFFERENT METABOLIZABLE ENERGY LEVELS AND FEED PROGRAMS AT 42 TO 57 DAYS OF AGE}

\section{ABSTRACT}

This experiment was conducted to evaluate the use of different energy levels and amino acid recommendations on performance, carcass yield and intestinal morphometry of broilers from 42 to 57 days of age. We used 1,600 oneday old male broilers (Cobb 500) in a completely randomized design arranged in a $2 \times 4$ factorial scheme with 3,200 and 3,600 kcal ME/kg and four different feed programs. The metabolizable energy levels and the feed programs did not determine significant differences in carcass characteristics for choosing a level of energy or a feed program, thus the energy level and the recommendations of amino acids that determine the highest cost-benefit ratio should prevail. It was observed that 3,600 kcal ME/ $\mathrm{kg}$ resulted in some improvement on performance and morphometry of the intestinal mucosa, also the fractioning of digestible amino acid requirements during two periods resulted in worse performance and intestinal villi height.

KEYWORDS: Digestible amino acids; metabolizable energy; nutritional requirements; small intestine. 


\section{INTRODUÇÃO}

A alta taxa de crescimento do frango de corte moderno é resultado, em grande parte, do melhoramento genético e das condições de produção, como nutrição e manejo (RAMOS et al., 2011). Com esse alto crescimento dos frangos de corte (RAMOS et al., 2009), houve uma maior valorização de rendimentos de cortes para posterior comercialização, sendo que esses cortes podem ser diretamente influenciados pela dieta e pelos programas alimentares (LIMA et al., 2008).

Durante muito tempo, prevaleceu a ideia de que a ave procura o alimento para satisfazer suas exigências em energia. No entanto, muitos trabalhos têm demonstrado que o controle do consumo de alimento é regulado pela densidade energética da ração (NASCIMENTO et al., 2011).

O desequilíbrio de componentes dietéticos, particularmente aminoácidos, limita o crescimento de tecido magro e direciona calorias para os adipócitos. O conteúdo energético também necessita de equilíbrio com os demais nutrientes, pois, em desequilíbrio, o excesso de energia favorecerá a deposição de gordura na carcaça (LESSON \& SUMMERS, 2001).

$O$ fato de que as exigências têm sido em grande parte determinadas em função do ganho de peso e da conversão alimentar, de certa, forma leva a uma dieta menos adequada para a maximização do crescimento de tecido magro. Porém, dentro do mesmo nível proteico, a suplementação de aminoácidos aumenta o conteúdo de proteína e reduz o teor de gordura do peito de frangos de corte (SI et al., 2001).

Com relação às possíveis alterações na composição da carcaça frente aos níveis de nutrientes, LIMA et al. (2008) concluíram que o nível energético de rações para frangos de corte afeta tanto o desempenho biológico quanto o econômico.

O bom desempenho das aves depende da obtenção adequada de energia e de compostos químicos pelo organismo. Para que isso ocorra, é necessário que o trato digestório apresente características estruturais e funcionais adequadas desde a ingestão dos alimentos até a sua absorção (CAMPOS et al., 2007). O elemento funcional do intestino delgado é a mucosa, que pode ser caracterizada como uma camada permeável a nutrientes e barreira contra compostos nocivos (OLIVEIRA et al., 2008).

$\mathrm{O}$ estudo da mucosa intestinal é um importante aspecto da fisiologia da digestão, pois representa uma extensa área de exposição a agentes exógenos que estão presentes nessa região a partir do início da ingestão, digestão e absorção de nutrientes (VIEIRA et al., 2006). A mucosa do intestino tem crescimento contínuo e é afetada por fatores exógenos e endógenos, como hormônios metabólicos, características físicas e químicas dos nutrientes e microrganismos presentes no intestino. A microbiota intestinal desempenha importante papel na manutenção da saúde animal (OLIVEIRA et al., 2008) e sua composição pode ser tanto benéfica quanto maléfica para o indivíduo, dependendo da natureza e da quantidade de microrganismos.

O número de vilosidades e seu tamanho, em cada segmento do intestino delgado, conferem a eles características próprias, sendo que na presença de nutrientes a capacidade absortiva do segmento será diretamente proporcional ao número de vilosidades ali presentes, tamanho dos vilos e área de superfície disponível para a absorção (MACARI et al., 2002). A absorção dos produtos da digestão ocorre inteiramente no intestino delgado, através de dois mecanismos, difusão e transporte ativo. Essa absorção é facilitada, além de outros fatores, pela disposição da mucosa em inúmeras projeções, chamadas vilosidades e invaginações da mucosa entre a base das vilosidades, formando as criptas de Lieberkhun (BALOG NETO et al., 2008).

Os objetivos deste estudo foram avaliar os efeitos de diferentes níveis de energia e programas de alimentação sobre o desempenho, as características de carcaça e a morfometria intestinal de frangos de corte de 42 e 57 dias de idade.

\section{MATERIAL E MÉTODOS}

Inicialmente, 1.600 pintos machos "Cobb 500 " de um dia de idade foram alojados em um galpão convencional de alvenaria, com cama de maravalha (aproximadamente cinco centímetros de altura). Nos períodos de 1 a 21 dias e de 22 a 41 dias de idade, todas as aves receberam as mesmas rações, formuladas para atender às exigências nutricionais, de acordo com ROSTAGNO et al. (2005). Ao final do $41^{\circ}$ dia de idade, todas as parcelas foram pesadas para assegurar peso médio semelhante no início do experimento (42 dias de idade).

Aos 42 dias de idade, 1.600 frangos de corte machos "Cobb 500" foram distribuídos em um delineamento inteiramente ao acaso, em esquema fatorial $2 \times 4$, perfazendo oito tratamentos com cinco repetições de 40 aves. Os fatores analisados foram dois níveis de energia (NE) metabolizável (3.200 e $3.600 \mathrm{kcal} \mathrm{EM} / \mathrm{kg}$ ) e quatro programas de alimentação (PA), compreendendo quatro recomendações de aminoácidos digestíveis, as quais variaram de acordo com a fonte de recomendação (POPE \& EMMERT, 2001; ROSTAGNO et al., 2005) e a fase de criação das aves (42 a 57 dias; 42 a 
49 dias e 50 a 57 dias de idade), representados no esquema abaixo:

- Programa alimentar 1 (PA1): ração formulada segundo as recomendações de ROSTAGNO et al. (2005), para lisina, metionina+cistina e treonina digestíveis, fornecida no período de 42 a 57 dias de idade;

- Programa alimentar 2 (PA2): ração formulada para conter lisina, metionina+cistina e treonina digestíveis, segundo a equação predita por POPE \& EMMERT (2001), fornecida no período de 42 a 57 dias de idade;

- Programa alimentar 3 (PA3): ração formulada para conter lisina, metionina+cistina e treonina digestíveis, segundo a equação predita por POPE \& EMMERT (2001), fornecida no período de 42 a 49 dias de idade, e ração PA4, fornecida no período de 50 a 57 dias de idade;

- Programa alimentar 4 (PA4): ração PA1, fornecida no período de 42 a 49 dias de idade e ração, formulada para conter lisina, metionina+cistina e treonina digestíveis, segundo a equação predita por POPE \& EMMERT (2001), fornecida no período de 50 a 57 dias de idade.

As equações de regressão de POPE \&
EMMERT (2001), elaboradas para atender às exigências de frangos machos dentro de cada fase de alimentação, foram:

- Metionina + Cistina digestível $=0,88-0,0063 \mathrm{x}$

- Lisina digestível $=1,22-0,0095 \mathrm{x}$

- Treonina digestível $=0,8-0,0053 \mathrm{x}$

$\mathrm{x}=$ idade média dentro do período desejado.

De acordo com a aplicação das equações de POPE \& EMMERT (2001), os níveis de metionina+cistina digestíveis foram de 0,57 (42 a 57 dias), 0,59 (42 - 49 dias) e 0,54 (50 a 57 dias); os de lisina digestível, de 0,75 (42 a 57 dias), 0,78 (42 a 49 dias) e 0,71 (50 a 57 dias); e os de treonina digestível, de 0,54 (42 a 57 dias), 0,56 (42 a 49 dias) e 0,51 ( 50 a 57 dias). As rações experimentais foram formuladas à base de milho, farelo de soja, óleo de soja, fosfato bicálcico, calcário calcítico, suplemento de vitaminas e microminerais (Tabela 1).

Ao final do período experimental, quando as aves atingiram 57 dias de idade, foram avaliados os dados de ganho de peso, consumo de ração, conversão alimentar, conversão calórica (kcal consumida/ $\mathrm{kg}$ de ganho de peso) e morfometria intestinal.

TABELA 1. Composição percentual e níveis nutricionais calculados das rações experimentais

\begin{tabular}{|c|c|c|c|c|c|c|c|c|}
\hline \multirow{3}{*}{ Ingredientes } & \multicolumn{8}{|c|}{ Rações experimentais } \\
\hline & \multicolumn{4}{|c|}{$3.200 \mathrm{kcal} \mathrm{EM} / \mathrm{kg}$} & \multicolumn{4}{|c|}{$3.600 \mathrm{kcal} \mathrm{EM} / \mathrm{kg}$} \\
\hline & PA1 & PA2 & PA3 & PA4 & PA1 & PA2 & PA3 & PA4 \\
\hline Milho grão & 64,50 & 64,85 & 64,89 & 65,04 & 55,02 & 55,50 & 55,46 & 55,55 \\
\hline Farelo de soja & 27,35 & 27,30 & 27,30 & 27,25 & 29,13 & 29,00 & 29,00 & 29,00 \\
\hline Óleo de soja & 4,61 & 4,55 & 4,50 & 4,43 & 12,34 & 12,19 & 12,20 & 12,17 \\
\hline Fosfato bicálcico & 1,43 & 1,43 & 1,43 & 1,44 & 1,45 & 1,45 & 1,45 & 1,45 \\
\hline Calcário calcítico & 0,91 & 0,92 & 0,91 & 0,92 & 0,89 & 0,89 & 0,90 & 0,90 \\
\hline Suplemento min + vit* & 0,50 & 0,50 & 0,50 & 0,50 & 0,50 & 0,50 & 0,50 & 0,50 \\
\hline Sal & 0,40 & 0,40 & 0,40 & 0,40 & 0,40 & 0,40 & 0,40 & 0,40 \\
\hline DL-Metionina $98 \%$ & 0,16 & 0,055 & 0,075 & 0,024 & 0,17 & 0,066 & 0,086 & 0,035 \\
\hline L-Lisina $\mathrm{HCl} 78 \%$ & 0,14 & - & - & - & 0,10 & - & - & - \\
\hline Total & 100,00 & 100,00 & 100,00 & 100,00 & 100,00 & 100,00 & 100,00 & 100,00 \\
\hline \multicolumn{9}{|l|}{$\overline{\text { Valores Calculados }}$} \\
\hline$\overline{\mathrm{EM}}\left(\mathrm{kcal} \mathrm{kg}^{-1}\right)$ & 3200 & 3200 & 3200 & 3200 & 3600 & 3600 & 3600 & 3600 \\
\hline Proteína Bruta (\%) & 18,00 & 18,00 & 18,00 & 18,00 & 18,00 & 18,00 & 18,00 & 18,00 \\
\hline Calcio (\%) & 0,80 & 0,80 & 0,80 & 0,80 & 0,80 & 0,80 & 0,80 & 0,80 \\
\hline Fósforo disponível (\%) & 0,37 & 0,37 & 0,37 & 0,37 & 0,37 & 0,37 & 0,37 & 0,37 \\
\hline Lisina digestível (\%) & 0,94 & 0,86 & 0,86 & 0,86 & 0,94 & 0,86 & 0,86 & 0,86 \\
\hline Met.+Cist. dig. (\%) & 0,67 & 0,57 & 0,59 & 0,54 & 0,67 & 0,57 & 0,59 & 0,54 \\
\hline Treonina digestível (\%) & 0,61 & 0,60 & 0,61 & 0,61 & 0,61 & 0,60 & 0,61 & 0,61 \\
\hline Sódio (\%) & 0,19 & 0,19 & 0,19 & 0,19 & 0,19 & 0,19 & 0,19 & 0,19 \\
\hline
\end{tabular}

*Enriquecido por quilograma de ração: Vitaminas: (A 8.000 UI; E 50 mg; D3 2.000 UI; K3 2,0 mg; B1 1,5 mg; B2 4,0 mg; B6 2,0 mg; B12 15,0 mcg); Ácido Pantotênico 12,0 mg; Ácido fólico 0,8 mg; Niacina 30,0 mg; Biotina 0,04 mg; Colina $200 \mathrm{mg}$; Cobre 8,0 mg; Iodo 1,0 mg; Selênio 0,3 mg; Manganês 70,0 mg; Zinco 80,0 mg; Ferro 50,0 mg; Antioxidante BHT 50,0 mg. 
Para avaliação das características de carcaça, quatro aves de cada parcela foram colhidas ao acaso, identificadas, pesadas individualmente e separadas em boxes, no qual permaneceram por um período de aproximadamente seis horas de jejum, recebendo apenas dieta hídrica, sendo posteriormente transportadas até $\mathrm{o}$ abatedouro onde foram novamente pesadas, procedendo-se o abate.

Após a evisceração, o frango passou por dois sistemas de banho em água gelada chamados préchiller e chiller, que preparam a carcaça para o resfriamento ou congelamento. Durante esse processo, a carcaça absorve água que congelará junto com o produto caso não seja realizado um gotejamento adequado. Após um período curto de gotejamento (permanecendo, portanto, uma maior quantidade de água retida na carcaça), as carcaças foram novamente pesadas sem pés, cabeça e pescoço, realizando-se então os cortes para avaliação dos seus respectivos rendimentos que consistiram em: peito, coxa + sobrecoxa e gordura abdominal.

No $57^{\circ}$ dia de experimento foi retirada uma amostra aleatória de duas aves de cada repetição, as quais foram pesadas e abatidas por decapitação, para escoamento do sangue. Procedeu-se à colheita de uma porção média do duodeno de aproximadamente quatro centímetros, a qual foi fixada por imersão em solução de Bouin por 24 horas. Os fragmentos dos tecidos foram destinados à rotina histológica com inclusão do material em paraplast. Após a microtomia, foram obtidos 15 cortes longitudinais e semi-seriados de sete micrômetros de espessura, corados pela técnica do Ácido Periódico de Schiff (PAS) e Hematoxilina e observados à microscopia de luz.

Foram realizadas as leituras de 30 medidas da altura das vilosidades e da profundidade das criptas intestinas (micrômetro), de cada tratamento, por meio de um analisador de imagens da Kontron Elektronik (Vídeo Plan), acoplado a um microscópio binocular. As medidas de altura de vilo foram tomadas a partir da região basal da mucosa intestinal, coincidente com a porção superior das criptas, até seu ápice. As criptas foram medidas da sua base até a região de transição cripta:vilosidade. Após a obtenção dos resultados, eles foram analisados estatisticamente.

As análises estatísticas foram realizadas utilizando-se o programa ESTAT (1994). Em caso de significância estatística, as médias foram comparadas pelo Teste de Tukey a $5 \%$ de probabilidade.

\section{RESULTADOS E DISCUSSÃO}

Na Tabela 2 são apresentadas as médias para os dados de desempenho na fase de 42 a 57 dias de idade. Não houve interação $(\mathrm{P}>0,05)$ entre os níveis de energia e programas de alimentação para o consumo de ração, ganho de peso e conversão alimentar. Foi observado efeito significativo $(\mathrm{P}<0,05)$ dos níveis de energia apenas para conversão alimentar, sendo o melhor resultado encontrado no nível de $3.600 \mathrm{kcal} \mathrm{EM} / \mathrm{kg}$.

TABELA 2. Consumo de ração (CR), ganho de peso (GP), conversão alimentar (CA) e conversão calórica (CC) das aves de acordo com os níveis de energia da ração (NE) e programas de alimentação (PA) e o desdobramento da interação NE $\times$ PA para CC, na fase de 42 a 57 dias de idade

\begin{tabular}{lcccc}
\hline Tratamentos & CR $(\mathrm{g})$ & GP $(\mathrm{g})$ & $\mathrm{CA}$ & $\mathrm{CC}(\mathrm{kcal} / \mathrm{g})$ \\
\hline \multicolumn{5}{c}{ Níveis de Energia } \\
\hline 3.200 & 3000 & 1088 & $2,76 \mathrm{~b}$ & 8,828 \\
3.600 & 2891 & 1118 & $2,59 \mathrm{a}$ & 9,107 \\
\hline
\end{tabular}

Programas de Alimentação

\begin{tabular}{llllrc}
\hline & & & & $3200 \mathrm{kcal}$ & $3600 \mathrm{kcal}$ \\
\hline PA1 & $2577 \mathrm{c}$ & 1127 & $2,29 \mathrm{a}$ & $7,715 \mathrm{Ca}$ & $6,938 \mathrm{Ba}$ \\
PA2 & $2529 \mathrm{c}$ & 1059 & $2,40 \mathrm{a}$ & $8,221 \mathrm{BCa}$ & $8,073 \mathrm{Ba}$ \\
PA3 & $3219 \mathrm{~b}$ & 1109 & $2,90 \mathrm{~b}$ & $9,145 \mathrm{ABb}$ & $10,625 \mathrm{Aa}$ \\
PA4 & $3456 \mathrm{a}$ & 1117 & $3,09 \mathrm{~b}$ & $10,228 \mathrm{Aa}$ & $10,789 \mathrm{Aa}$ \\
\hline
\end{tabular}

Valores de F

\begin{tabular}{lcccc}
\hline $\mathrm{NE}$ & $3,6479^{\mathrm{NS}}$ & $1,6407^{\mathrm{NS}}$ & $6,3799^{*}$ & $1,5265^{\mathrm{NS}}$ \\
$\mathrm{PA}$ & $65,9570^{* *}$ & $1,7039^{\mathrm{NS}}$ & $33,6509^{* *}$ & $43,0051^{* *}$ \\
$\mathrm{NE} x \mathrm{PA}$ & $1,1542^{\mathrm{NS}}$ & $0,6883^{\mathrm{NS}}$ & $1,8672^{\mathrm{NS}}$ & $4,6038^{*}$ \\
\hline $\mathrm{CV}(\%)$ & 5,48 & 5,91 & 7,07 & 7,12 \\
\hline
\end{tabular}

Médias na coluna seguidas de letras maiúsculas e na linha seguidas de letras minúsculas diferentes são significativas pelo teste de Tukey $(\mathrm{P}<0,05)$. ** $\mathrm{P}<0,01 ; * \mathrm{P}<0,05 ; \mathrm{NS}=$ não significativo. 
Não foram observados efeitos significativos $(\mathrm{P}>0,05)$ dos níveis de energia sobre o consumo de ração e ganho de peso no presente estudo. LIMA et al. (2008), em estudo com diferentes níveis de energia $(2.900,3.000$ e $3.100 \mathrm{kcal} \mathrm{EM} / \mathrm{kg}$ ), lisina e metionina+cistina, observaram que o menor consumo de ração foi obtido com o menor nível de energia $(\mathrm{P}<0,05)$. É sabido que o maior consumo pode estar associado ao maior nível de óleo nas rações de média e alta energia, o que aumenta a palatabilidade, favorecendo a ingestão e aumento do consumo de ração. CELLA et al. (2009), estudando diferentes níveis de lisina $(1,14 ; 1,18 ; 1,22$ e $1,26 \%$ de lisina digestível), verificaram que houve efeito quadrático $(\mathrm{P}<0,05)$ sobre o ganho de peso das aves, sendo o melhor nível encontrado de $1,18 \%$ de lisina digestível.

BARBOSA et al. (2008), trabalhando com diferentes níveis de energia e temperatura ambiente, obtiveram resposta semelhante à encontrada neste estudo para ganho de peso, sendo que essa variável também não foi influenciada pelos níveis de energia metabolizável da ração.

Os programas de alimentação exerceram efeito significativo $(\mathrm{P}<0,01)$ sobre o consumo de ração e conversão alimentar, sendo os menores consumos e melhores valores de conversão alimentar proporcionado pelos programas PA1 e PA2.

Para conversão calórica ocorreu interação significativa $(\mathrm{P}<0,05)$ entre os fatores estudados, sendo o desdobramento apresentado na Tabela 2. Dentro do nível de $3.200 \mathrm{kcal} \mathrm{EM} / \mathrm{kg}$ de ração, observou-se que a melhor conversão calórica foi obtida com o programa de alimentação PA1 e as piores com os programas PA3 e PA4. Já no nível de $3.600 \mathrm{kcal} \mathrm{EM} / \mathrm{kg}$ de ração, não foi encontrada diferença entre os programas PA1 e PA2 e entre os programas PA3 e PA4. No entanto, PA1 e PA2 foram diferentes de PA3 e PA4, sendo os melhores resultados encontrados para os programas PA1 e PA2.

Dessa forma, os programas PA1 e PA2, adotando as recomendações de aminoácidos de ROSTAGNO et al. (2005) ou aplicando as equações de POPE \& EMMERT (2001) para determinar os níveis de aminoácidos, considerando-se um único nível de aminoácidos para a fase de 42 a 57 dias, proporcionaram desempenhos semelhantes $\mathrm{e}$ melhores em relação aos demais programas.

Na Tabela 3, são apresentados os dados de rendimento de carcaça de peito e de coxa + sobrecoxa e teor de gordura abdominal.

TABELA 3. Rendimento de carcaça (RC), de peito (RP), de coxa + sobrecoxa (RC+SC) e gordura abdominal (GA) de frangos de corte abatidos aos 57 dias de idade das aves de acordo com os níveis de energia da ração (NE) e programas de alimentação (PA)

\begin{tabular}{|c|c|c|c|c|}
\hline Tratamentos & $\mathrm{RC}^{* 1}(\mathrm{~g})$ & RP (g) & $\mathrm{RC}+\mathrm{SC}(\mathrm{g})$ & GA $(\%)$ \\
\hline \multicolumn{5}{|c|}{ Nível de energia (NE) } \\
\hline$\overline{3.200}$ & 81,28 & 34,10 & 35,70 & 2,00 \\
\hline 3.600 & 81,21 & 34,50 & 35,25 & 2,14 \\
\hline \multicolumn{5}{|c|}{ Programas de alimentação (PA) } \\
\hline$\overline{\mathrm{PA} 1}$ & 81,81 & 34,79 & 35,41 & 2,16 \\
\hline PA2 & 81,68 & 33,91 & 35,52 & 2,25 \\
\hline PA3 & 81,36 & 34,27 & 35,54 & 2,15 \\
\hline PA4 & 81,14 & 34,23 & 35,43 & 1,73 \\
\hline \multicolumn{5}{|l|}{$\overline{\text { Valores de } \mathrm{F}^{1}}$} \\
\hline$\overline{\mathrm{NE}}$ & $0,0093^{\mathrm{NS}}$ & $0,8070^{\mathrm{NS}}$ & $1,2802^{\mathrm{NS}}$ & $0,4199^{\mathrm{NS}}$ \\
\hline PA & $0,2665^{\mathrm{NS}}$ & $0,6377^{\mathrm{NS}}$ & $0,0260^{\mathrm{NS}}$ & $1,1722^{\mathrm{NS}}$ \\
\hline NE $x$ PA & $1,4585^{\mathrm{NS}}$ & $2,7194^{\mathrm{NS}}$ & $1,6640^{\mathrm{NS}}$ & $0,5362^{\mathrm{NS}}$ \\
\hline$\overline{\mathrm{CV}}(\%)$ & 2,78 & 3,69 & 3,25 & 29,44 \\
\hline
\end{tabular}

**P< 0,$01 ; * \mathrm{P}<0,05 ; \mathrm{NS}=$ não significativo;

${ }^{1}$ carcaças evisceradas, sem pés, sem cabeça e sem pescoço, pesadas logo após o chiller.

Constatou-se que não houve interação significativa entre os fatores estudados e nem efeito isolado dos mesmos sobre as características avaliadas $(\mathrm{P}>0,05)$. Esses resultados concordam com o de LIMA et al. (2008) em que o rendimento de carcaça dos machos não apresentou interações
( $\mathrm{P}>0,05)$ entre os níveis energéticos e níveis de aminoácidos (lisina e metionina+cistina) das dietas experimentais. SUMMERS et al. (1988), em trabalho com níveis de 0,73; 0,93 e 1,03\% de lisina, também não encontraram diferenças significativas para rendimento de carcaça em aves alimentadas com 
diferentes níveis de lisina e metionina+cistina na fase final de criação. SILVIA FILHA et al. (2004), em estudo com diferentes níveis energéticos da ração, encontraram maior rendimento de carcaça $(81,28 \%)$ com o nível $3.150 \mathrm{kcal}$ de EM/kg.

Com relação à gordura abdominal, não foram observados efeitos significativos $(\mathrm{P}>0,05)$ entre os níveis de energia estudados ou programas de alimentação, discordando dos achados de BARBOSA et al. (2008) em que as porcentagens de peito e de gordura abdominal das aves foram afetadas $(\mathrm{P}<0,01)$ pelos níveis de energia da ração, sendo esses níveis 2.800, 2.900, 3.000, 3.100 e 3.200 $\mathrm{kcal} \mathrm{EM} / \mathrm{kg}$, respectivamente.

Na Tabela 4 são apresentados os resultados de morfometria do duodeno dos frangos de corte abatidos aos 57 dias de idade e o desdobramento da interação significativa entre os níveis de energia e programas de alimentação para altura de vilosidade $(\mathrm{P}<0,05)$. O nível de $3.200 \mathrm{kcal} \mathrm{EM} / \mathrm{kg}$ e programa PA1 apresentaram menor altura de vilosidade. Esse resultado concorda com os encontrados por
PELICANO et al. (2003) que relataram menor altura de vilosidade para $3.200 \mathrm{kcal} \mathrm{EM} / \mathrm{kg}$ e $0,935 \%$ de lisina na ração. Da mesma forma, ANDRADE et al. (2004), em seu estudo em que avaliou silagem de grãos úmidos de milho e sua associação com aditivos nas rações, mostraram que a menor altura de vilosidade foi encontrada no nível $3.202 \mathrm{kcal} \mathrm{EM} / \mathrm{kg}$ para o tratamento controle. Porém, esses resultados divergem dos encontrados por BALOG NETO et al. (2008) que não encontraram efeito da energia sobre a altura da vilosidade.

Por outro lado, no nível $3.600 \mathrm{kcal} \mathrm{EM} / \mathrm{kg}$, as recomendações de aminoácidos não determinaram diferenças na altura de vilosidade $(\mathrm{P}>0,05)$.

Em relação ao nível de energia, observou-se, com exceção do programa PA1, que o nível 3.600 $\mathrm{kcal} \mathrm{EM} / \mathrm{kg}$ proporcionou maior altura de vilosidade intestinal. Dentre outros fatores, as características da mucosa do intestino, como altura de vilosidade, são afetadas por características físicas e químicas dos nutrientes, o que pode ser evidenciado pelo maior nível de energia.

TABELA 4. Altura de vilosidade (HV), profundidade de cripta (PC) e relação HV/PC do duodeno de frangos de corte abatidos aos 57 dias de idade das aves de acordo com os níveis de energia da ração (NE) e programas de alimentação (PA) e o desdobramento da interação $\mathrm{NE} \times \mathrm{PA}$ para $\mathrm{HV}$ aos 57 dias de idade

\begin{tabular}{lccc}
\hline \multirow{2}{*}{ Tratamentos } & HV $(\mu \mathrm{m})$ & $\mathrm{PC}(\mu \mathrm{m})$ & HV/PC \\
\cline { 2 - 4 } & & Níveis de Energia $(\mathrm{NE})$ & 3254,40 \\
3.200 & 1246,30 & 403,60 & 3404,40 \\
\hline
\end{tabular}

Programas de alimentação (PA)

\begin{tabular}{|c|c|c|c|c|}
\hline & $3.200 \mathrm{kcal}$ & $3.600 \mathrm{kcal}$ & & \\
\hline PA1 & $1,1210 \mathrm{Ab}$ & $1,4553 \mathrm{Aa}$ & $471,10 \mathrm{~A}$ & $2884,30 \mathrm{~B}$ \\
\hline PA2 & $1,3818 \mathrm{Aa}$ & $1,2675 \mathrm{Aa}$ & $346,60 \mathrm{~B}$ & $3935,10 \mathrm{~A}$ \\
\hline PA3 & $1,2060 \mathrm{Aa}$ & $1,2663 \mathrm{Aa}$ & $400,80 \mathrm{AB}$ & $3176,60 \mathrm{AB}$ \\
\hline \multirow[t]{2}{*}{ PA4 } & $1,2765 \mathrm{Aa}$ & $1,2803 \mathrm{Aa}$ & $411,50 \mathrm{AB}$ & $3321,80 \mathrm{AB}$ \\
\hline & \multicolumn{4}{|c|}{ Valores de F } \\
\hline F NE & \multicolumn{2}{|c|}{$1.8790^{\mathrm{NS}}$} & $0.1219^{\mathrm{NS}}$ & $0.4234^{\mathrm{NS}}$ \\
\hline F PA & \multicolumn{2}{|c|}{$0.4935^{\mathrm{NS}}$} & $5.2863 * *$ & $3.6908 *$ \\
\hline F NEXPA & \multicolumn{2}{|c|}{$3.3626 *$} & $1.3042^{\mathrm{NS}}$ & $1.5946^{\mathrm{NS}}$ \\
\hline $\mathrm{CV}(\%)$ & \multicolumn{2}{|c|}{11,43} & 15,41 & 19,58 \\
\hline
\end{tabular}

Médias na coluna seguidas de letras maiúsculas e na linha seguidas de letras minúsculas diferentes são significativas pelo teste de Tukey $(\mathrm{P}<0,05)$. ** $\mathrm{P}<0,01 ; * \mathrm{P}<0,05 ; \mathrm{NS}=$ não significativo.

Não houve efeito significativo $(\mathrm{P}>0,05)$ dos níveis de energia testados sobre os parâmetros de profundidade de cripta (PC) e relação altura de vilo/profundidade de cripta (HV/PC). Esses resultados concordam com os achados de BALOG NETO et al (2008) e ANDRADE et al. (2004), que, da mesma forma, não encontraram efeito da energia da dieta sobre a profundidade de cripta e relação de vilo/profundidade; no entanto, a profundidade de cripta tendeu a ser maior numericamente de acordo com a dieta com maior porcentagem de energia.

Em relação aos programas de alimentação, 
os melhores valores de profundidade de cripta (PC) foram obtidos com o programa PA1, embora não tenha diferido dos programas PA3 e PA4. Esses resultados discordam dos achados de PELICANO et al (2003), que verificaram valores inferiores aos do presente estudo para profundidade de cripta para todos os tratamentos. O epitélio intestinal das aves está em constante renovação através de mecanismos de desenvolvimento e reparo da mucosa intestinal (turnover), os quais podem ser alterados sob ação de agentes patogênicos ou nutricionais, tornando-se hiperplástico, com maior profundidade de cripta (CAMPOS et al., 2007).

Para a relação altura de vilo/profundidade de cripta (HV/PC), os melhores valores foram obtidos para o programa PA2, sendo que o mesmo não diferiu dos programas PA3 e PA4.

O desenvolvimento da mucosa intestinal consiste no aumento da altura e densidade dos vilos, o que corresponde ao aumento das células epiteliais, conferindo uma melhor digestão e absorção intestinal (CAMPOS et al., 2007), como foram evidenciados pelo maior nível de energia, que proporcionou maior altura de vilosidade e maior desempenho aos animais.

\section{CONCLUSÕES}

O maior nível energético e os programas de alimentação PA1 e PA2 proporcionaram alguma melhoria no desempenho das aves, enquanto que o fracionamento das exigências de aminoácidos digestíveis em dois períodos, como nos programas PA3 e PA4, determinou piores resultados.

Os níveis energéticos ou os diferentes programas de alimentação não determinam diferenças expressivas na qualidade da carcaça que justificassem a escolha de um nível ou de um programa alimentar, devendo, portanto, prevalecer o nível energético e as recomendações de aminoácidos que determinem o melhor custo-benefício.

O maior nível energético $(3.600 \mathrm{kcal}$ $\mathrm{EM} / \mathrm{Kg}$ ), com exceção do programa de alimentação 1 (PA1), proporcionou melhores resultados para altura de vilosidade.

\section{AGRADECIMENTO}

À FAPESP pelo Auxílio Financeiro concedido proc. 2002/10791-0.

\section{REFERÊNCIAS}

ANDRADE, R. C.; SARTORI, J. R.; GONÇALVES, J.
C.; MARTINEZ, K. L. A.; COSTA, C.; PEZZATO, C. A.; OLIVEIRA, H. N. Silagem de grãos úmidos de milho e aditivos na alimentação de frangos de corte. Acta Scientiarum Animal Sciences, v.26, n.4, p.553-559, 2004.

BALOG, A.; MENDES, A. A.; PAZ, I. C. de L. A.; TAKAHASHI, S. E.; GARCIA, R. G.; KOMIYAMA, C. M.; MARTINS, M. R. F. B.; BUENO, L. G. F. Morfometria do eptélio intestinal de frangos de corte tipo colonial alimentados com silagem de grãos úmidos de milho. PUBVET - Publicações em Medicina Veterinária e Zootecnia, v. 2, p. 248-248, 2008.

BARBOSA, F. J. V.; LOPES, J. B.; FIGUEIREDO, A. V.; ABREU, M. L. T.; DOURADO, L. R. B.; FARIAS, L. A.; PIRES, J. E. P. Níveis Energia Metabolizável em Rações de Frangos de Corte Mantidos em Ambiente de Alta Temperatura. Revista Brasileira de Zootecnia, v. 37, p. 849-855, 2008.

CAMPOS, D. M. B.; F. FILHO, D. E.; TORRES, K. A. A.; FURLAN, R. L.; MACARI, M. . Desenvolvimento da mucosa intestinal e a substituição do milho por sorgo na dieta de pintainhos de corte. Revista de Ciências Veterinárias, v. 5, p. 44-48, 2007.

CELLA, P. S.; MURAKAMI, A. E.; GALLI, R. J. Níveis de lisina digestível em dietas baseadas no conceito de proteína ideal para frangos de corte na fase inicial. Ciência Animal Brasileira, v. 10, p. 101-106, 2009.

ESTAT - SISTEMA DE ANÁLISES ESTATÍSTICAS. Universidade Estadual Paulista "Júlio de Mesquita Filho". Departamento de Ciências Exatas. ESTAT. Versão 2.0. Jaboticabal: FCAV/UNESP, 1994.

LESSON, S.; SUMMERS, J.D. Nutrition of the chicken. 4. ed. Guelph: University Books, p. 413, 2001.

LIMA, L. M. B.; LARA, L. J.; BAIAO, N. C.; CANCADO, S. V.; MICHELL, B. C.; FERREIRA, F. C. . Efeitos dos níveis de energia, lisina e metionina + cistina sobre o desempenho e rendimento de frangos de corte. Revista da Sociedade Brasileira de Zootecnia, v.37, n. 8, p. 1424-1432, 2008.

MACARI, M.; FURLAN, R. L.; GONZALES, E. Fisiologia Aviária Aplicada a Frangos de Corte. 2 ed Ampliada. Jaboticabal: editora FUNEP/UNESP, 2002, 375 p., capítulo 8, p. 113-123, 2002.

NASCIMENTO, G. A. J.; RODRIGUES, P. B.; FREITAS, R. T. F. ; REIS NETO, R. V.; LIMA, R. R.; ALLAMAN, I. B. Equações de predição para estimar valores da energia metabolizável de alimentos concentrados energéticos para aves utilizando a metaanálise. Arquivo Brasileiro de Medicina Veterinária e Zootecnia, v. 63, p. 222-230, 2011.

OLIVEIRA, M. C.; MARQUES, R. H.; GRAVENA, R. A.; MORAES, V. M. B. Morfometria do intestino delgado de frangos tratados com dietas adicionadas de mananoligossacarídeo e complexo enzimático. Revista Biotemas, v. 21, p. 135-142, 2008. 
PELICANO, E. R. L.; SOUZA, P. A.; SOUZA, H. B. A.; OBA, A.; NORKUS, E. A.; KODAWARA, L. M.; LIMA, T. M. A. Morfologia intestinal de frangos de corte alimentados com dietas contendo diferentes probióticos. Revista Portuguesa de Ciências Veterinárias, v. 98, n. 547, p. 125-134, 2003.

RAMOS, K. C. B. T.; GOMES, A. V. C.; CAMARGO, A. M.; LIMA, C. A. R.; OLIVEIRA, E. C. D.; OLIVEIRA, A. A.; SANTOS, V. M.; MASSI, P. A. Características da carcaça e composição química dos cortes nobres de frangos de corte submetidos a programas de restrição alimentar. Revista Ciências da Vida, v. 29, n. 1, p. 28-38, 2009.

RAMOS, K. C. B. T.; GOMES, A. V. da C.; LIMA, C. A. R. de; CAMARGO, A. M.; SILVA, R. V. M.; CURVELlO, F. A.; MASSI, P. de A. Desempenho produtivo e econômico de frangos de corte submetidos a programas de restrição alimentar. Ciência Animal Brasileira, v. 12, n. 1, p. 8-16, 2011.

ROSTAGNO, H. S.; ALBINO, L. F. T; DONZELE, J. L.; GOMES, P. C.; OLIVEIRA, R. F. M. de; LOPES, D. C.; FERREIRA, A. S.; BARRETO, S. L. T. Tabelas brasileiras para aves e suínos: composição de alimentos e exigências nutricionais. Viçosa, MG:
Universidade Federal de Viçosa, 2005. 186p.

SI, J.; FRITTS, C. A.; BURNHAM, D. J. .Relationship of dietary lysine level to the concentration of all essential amino acids in broiler diets. Poultry Science, v.80, n.10, p.1472-1479, 2001.

SILVA FILHA, O. L.; BARBOZA, W. A.; FARIAS FILHO, R. V.; RABELLO, C. B. V.; DUTRA JÚNIOR, W. M.; OLIVEIRA, R. J. F. . Efeito do nível energético da ração sobre o desempenho de frangos de corte no período de um a 21 dias de idade. Revista da Faculdade de Zootecnia, Veterinária e Agronomia, v. 11, n. 01, p. 0720, 2004.

SUMMERS, J. D.; LEESON, S., SPRATT, D. Yield and composition of edible meat from male broilers as influenced by dietary protein level and amino acid supplementation. Canadian Journal Animal Science, v. 68 , p. 241-248, 1988.

VIEIRA, B. S.; FARIA FILHO, D. E.; TORRES, K. A. A.; CAMPOS, D. M. B.; ROSA, P. S.; FURLAN, R. L. Administração in ovo de glutamina e de lisina sobre o desenvolvimento da mucosa intestinal de frangos na primeira semana pós-eclosão. ARS Veterinária, v. 22, p. 170-175, 2006.

Protocolado em: 04 maio 2010. Aceito em: 03 abr. 2012. 